\title{
DIETRICH SCHUBERT
}

\section{Nietzsches Blick auf Delacroix als Künstlertypus}

„Die Kunst ist das große Stimulans zum Leben“

(Götzendämmerung, Streifzüge 24)

Die Entscheidung für dieses Thema ist aus bestimmten Gründen erfolgt, die mit der zentralen Stellung von Nietzsches Kunstbegriff in seinem Denken und mit der neueren Problematik der Nietzsche-Rezeption in der heutigen Kunsthistorie gekoppelt sind. ${ }^{1}$

Bislang verharrte die Kunsthistorie bei den schwierigen Fragen, wie die verworrenen und weitverzweigten und teils nur als Fermente und gebrochen wirkenden Nietzsche-Impulse gefaßt, belegt und interpretiert werden können - insbesondere dann, wenn es keine konkreten Briefstellen oder Tagebücher gibt, d. h. der Dichter und Künstler ohne Rechenschaft irgend eine Nietzsche-Idee verarbeitete. Die Literaturhistorie war dabei schon seit vielen Jahren der Kunsthistorie voraus (z. B. W. Taraba, B. Hillebrand, G. Martens u. a.). ${ }^{2}$

Klaus Herdings Abhandlung von 1992 zu Picassos Demoiselles hat das Feld mit dem Blick auf Barcelona der „Generacion 98“ und auf das Paris von um 1904 (das Jahr der französischen Nietzsche-Ausgabe, Picasso siedelte nach Paris über, der Zarathustra war freilich schon 1898 in Paris erschienen, übers. v. Henri Albert) erweitert und Thesen von Ron Johnson und Marc Rosenthal (1980) modifiziert. - Noch 1913 hatte Apollinaire Nietzsche als dionysischen Interventen in Paris genannt. Dabei ist jedoch der Kontrast zwischen

1 Als Vortrag gehalten auf der von Klaus Herding organisierten Tagung an der Universität Frankfurt a.M. „Nietzsche und die Künste“, am 4.11.1994. Dabei spielte die Einleitung von H. D. Erbsmehl in seine jüngste Untersuchung der Nietzsche-Impulse im frühen 20. Jahrhundert auch eine gewisse Rolle: Kulturkritik und Gegenästhetik - zur Bedeutung Nietzsches für die bildende Kunst in Deutschland 1892-1918, Ann Arbor 1993, 22-48. D. Schubert, „Nietzsche-Konkretionsformen in der bildenden Kunst - ein Überblick“, in: Nietzsche-Studien 10/11 (1982/83), 278-317, mit einer grundsätzlichen Einleitung bes. zu G. Lukács und R. Hamann/J. Hermand; J. Krause, „Märtyrer “ und „Prophet “ - Studien zum Nietzschekult, Berlin 1984.

2 W. Taraba, „Der schöpferische Einzelne und die Gesellschaft in Nietzsches Zarathustra“, in: Literatur und Gesellschaft, Bonn 1963, 196 f.; B. Hillebrand, Artistik und Auftrag - zur Kunsttheorie von Benn und Nietzsche, München 1966; B. Hillebrand (Hg.), Nietzsche und die deutsche Literatur, 2 Bde., Tübingen 1978; G. Martens, Vitalismus und Expressionismus, Stuttgart 1971; G. Martens, „Nietzsches Wirkung im Expressionismus“, in: B. Hillebrand (Hg.), Nietzsche und die deutsche Literatur, 35-82; S. Vietta u. H. G. Kemper, Expressionismus, München 1975, 134 f.; H. G. Kemper, Vom Expressionismus zum Dadaismus, Kronberg 1974; S. Vietta, Lyrik des Expressionismus, 2. Aufl., München 1985, $16 \mathrm{f}$. (Hinfall der kosmologischen Werte). 
der Blauen Periode Picassos und der Dissoziation der Formen im Primitivismus nach 1906 scheinbar unüberbrückbar, - ein Bruch, wie er für die Krisen innerhalb der „Moderne“ nur allzu charakteristisch ist.

Die Quellen des Gestaltens, die tiefer als die jeweilige Form liegen, scheinen das eigentliche Movens zu sein; zu sehr hält sich Kunsthistorie oft an die Formen statt nach den Wurzel-Gründen derselben zu fragen, wie bereits 1932 Carl Einstein appellierte. ${ }^{3}$ Von den genannten Autoren wurde der enorme Bruch bzw. Schock im Wandel von der süßlichsentimentalen Periode Picassos bis 1905 zum Primitivismus ab 1906 mit seiner Formdominanz aus der Sprache der Negermasken dargestellt. ${ }^{4}$

War Nietzsches Wirkung subjektiv beliebig oder verstand Picasso erst gegen 1907 Nietzsches Formel von der (auch künstlerischen) „Umwertung aller Werte “ über den ICH-Kult der frühen Jahre hinaus - nun als Appell und Kunst-Problem? - beziehungsweise wie Gottfried Benn meinte: „Wie ist Gestaltung (noch) möglich? “ Benn antwortete (1955): „Gestaltung, das war kein artistischer Begriff, sondern hieß: Was für ein Rätsel, was für ein Geheimnis, daß der Mensch Kunst macht, daß er der Kunst bedürftig ist, - was für ein einziges Erlebnis innerhalb des europäischen Nihilismus! " Hier sprach quasi Nietzsche aus Benns Mund. ${ }^{5}$

Ich selbst habe den Fragen der Wirkung der emanzipatorischen Ideen Nietzsches - gegenüber dem quantitativ breiteren panegyrischen Nietzsche-Kult um und nach 1900 - bewußt Priorität eingeräumt, weil jede Geschichtsschreibung dem „atmenden Heute“ entspringt (C. Einstein). ${ }^{6}$ Dies wurde zum Teil kritisiert, zum Teil jedoch auch nicht verstanden. Aber gerade der wesentlich schmalere Teil der demokratisch, europäisch und emanzipatorisch (anti-wilhelminisch) ${ }^{7}$ orientierten Nietzsche-Anhänger und Leser vor und während des 1. Weltkriegs scheint mir im Hinblick auf Albert Camus' Nietzsche-Rezeption und hinsichtlich unserer Zeit und unseres Selbstverständnisses bedeutender. ${ }^{8}$

Wirkungen bzw. Impulse habe ich thematisiert für den frühen Max Beckmann: Auferstehung als ein Schweben Gleicher zum Licht (1909), für den sozialkritisch radikalen Feststel-

3 Als Beispiel formaler Kunsthistorie s. P. A. Riedl, Kandinsky, Reinbek 1982, der sogar Einsteins Kandinsky-Kapitel in der Propyläen-Kunstgeschichte verschweigt; C. Einstein, Die Kunst des 20. Jahrhunderts [1926], 3. Aufl., Berlin 1931, 204: Kandinskys Malerei „ein Mittel geistiger Isolierung gegen die Welt". Carl Einstein blickte auf „die Bedingungen, woraus die Kunstwerke erwachsen": Georges Braque, Paris 1934, dt. Typoskript im Einstein-Archiv Berlin, inzwischen publiziert in C. Einstein, Werke, Bd. 3, hg. v. M. Schmid u. L. Meffre, 1985, 192.

4 M. Rosenthal, The nietzschean character of Picasso's early development, in: Arts Magazine 55 (1980), 87-91; R. Johnson, The Demoiselles d'Avignon and dionysian destruction, in: Arts Magazine 55 (1980), 94-101 f., 102 f.; Klaus Herding, Picasso - Les Demoiselles d'Avignon, Frankfurt a.M. 1992, 80 f.; G. Apollinaire, Les peintres cubiste, Paris 1913, 16 (dt. Zürich 1956, 25). Der Zarathustra erschien in Paris schon 1898 in Henri Alberts Übersetzung. Für die frühe französische Nietzsche-Rezeption H. Lichtenberger, La philosophie de Nietzsche, Paris 1898 (dt. Dresden 1899); dazu J. Le Rider, Nietzsche in Frankreich (Paris 1993), München 1997, $53 \mathrm{f}$.

5 G. Benn, Einleitung, Lyrik des expressionistischen Jahrzehnts, München 1955, 10, 13.

6 C. Einstein, „Antike und Moderne" (Nachlaßtext), in: Existenz und Ästhetik, hg. v. S. Penkert, Wiesbaden 1970, 44.

7 Hierin treffe ich mich in jüngerer Zeit mit R. Reschke, Nietzsche, Fröhliche Wissenschaft, Edition Leipzig 1990, 369.

8 A. Camus, „Nietzsche und der Nihilismus“, in: L'Homme revolté, Paris 1951, dt. Reinbek 1969, 55-67, wiederabgedruckt in: J. Salaquarda (Hg.), Nietzsche, Darmstadt 1980, $63 \mathrm{f}$. 
lungswillen von Otto Dix ab 1920, für den Emporsteigenden des Bildhauers Wilhelm Lehmbruck von 1914 (1916 ausgestellt) und für das synthetische Projekt Bruno Tauts von 1919/20, Monument des neuen Gesetzes, das Ideen amalgamiert aus Luther, Karl Liebknecht, dem Propheten Haggai und aus der Johannes-Apokalypse mit Nietzsches radikaler Staatskritik in Vom neuen Götzen 1884 im Zarathustra: „Dort wo der Staat aufhört, da beginnt erst der Mensch, der nicht überflüssig ist ... Dort wo der Staat aufhört - so seht mir doch hin, meine Brüder! Seht ihr ihn nicht, den Regenbogen und die Brücken des Übermenschen? -"

Zur Zeit dreht sich die Bemühung, Nietzsches Impulse in den bildenden Künsten zu fassen, teilweise im Kreise, teils wurde sie ängstlich unterlassen (so von den Kandinsky- und Marc-Bearbeitern) oder mangelhaft gestreift (wie in Whytes Buch über B. Taut 1981); neues Material stellt H. Erbsmehl vor betreffs Melchior Lechters Nietzschekult und für die expressionistische Kunst um Marc und Burger im Kriege. ${ }^{10}$

Im Katalog $O$ meine Zeit ... von 1985 hatte Roland Bothner in der Studie Das zweite Gesicht des Willens die Nietzsche-Fermente in Marcs Schriften und Programmkunst genauer untersucht - nach 1. der metaphysischen Verfassung der Kunst, 2. der „Annahme der Differenz von Wesen und Erscheinung “ und 3. der metaphysischen Begründung der "Tätigkeit des Künstlers".

Wie sich in meiner Perspektive zeigen wird, ist Kunstschaffen für Nietzsche jedoch immer Emotion, Feiern, Loben, Gestaltung des wirklichen Lebens. Franz Marcs Postulat aber, der Künstler solle „nicht das Lob unseres häßlichen Seins singen, sondern unseres Dryadenwillens zum Anderssein", war - wie Bothner übersah - eine totale Wende Marcs weg von Nietzsches Position hin zur Spaltung der Welt, zur Leugnung und Abwertung der wirklichen Welt, die Nietzsche leidenschaftlich als das Einzige feierte (Geschichte eines Irrtums in Götzendämmerung). ${ }^{11}$

9 ZA, „Vom neuen Götzen“, KGW VI/1, 57-60; D. Schubert, in: Jahrbuch der Berliner Museen 29/30 (1987/88), 241-255, zu Bruno Taut und Nietzsche. Zu W. Lehmbruck vgl. meine Monographie Die Kunst Lehmbrucks, 2. verb. Aufl., Dresden 1990, 183; zu Dix vgl. meine Rowohlt-Monographie, 3. Aufl., Reinbek 1991, 54 f.; und O. Conzelmann, Der andere Dix, Stuttgart 1983, 211 f. Das Ausweichen vor der Nietzsche-Frage wie für Kandinsky bei P. A. Riedl betonte jetzt für Franz Marc auch H. D. Erbsmehl, Kulturkritik, 295; der Brief Marcs vom 21.1.1911 wurde immer nur abgedruckt, s. R. Gollek, Franz Marc, München 1980, 114.

10 Es ist hier nicht nötig, alle die Studien aufzulisten über die Nietzsche-Impulse von G. Fechter, G. Svenaeus, G. Stamm, T. Buddensieg, G. Reinhardt (zur Brücke), E. G. Güse (zu Beckmann), G. Schuster (im Kat. Graf Kessler 1988), J. Block, J. Krause 1984, D. Teubner (über H. van de Velde 1984, H. D. Erbsmehl, H. Adam (Diss. 1996 in Heidelberg) u. a. Hinweisen möchte ich eigens auf Kurt Badt, in dessen kunsttheoretischen Schriften Nietzsches Kunstbegriff appliziert wurde; K. Badt, Kunsttheoretische Versuche, hg. v. L. Dittmann, Köln 1968, 85 f., 114 f. - die Wirkung Nietzsches auf Bruno Taut als Baumeister utopischer Projekte und seinen Kollegenkreis der "Gläsernen Kette“ 1919-1920 nur mangelhaft berücksichtigt bei J. B. Whyte, Bruno Taut, Stuttgart 1981, 146. Für Paris und Picasso vor 1907 K. Herding, Picasso, 77; Nietzsche war das Leitbild der „Generation von 98“ (s. Anm. 4); H. D. Erbsmehl, Kulturkritik, Kap. 6, 286 f.

11 Nietzsche entlarvte die Spaltung in eine wahre und eine scheinbare Welt, eine Spaltung, der Marc letztlich ganz verhaftet blieb; F. Marc, Schriften, Köln 1978, 212. R. Bothner, „Das zweite Gesicht des Willens", in: O meine Zeit - so namenlos zerrissen (Kat. d. Ausst. v. J. Hülsewig-Johnen), Bielefeld 1985, 62-76. Bereits Klaus Lankheit hatte die Wendung Marcs von Nietzsches Weltsicht zu Schopenhauers Verneinung des Willens zum Leben konstatiert; K. Lankheit, F. Marc - sein Leben und 
Mir selbst wird nach vielen Jahren ein Gedanke immer wesentlicher, nämlich ob Teile von Nietzsches Existenz-Philosophie der Priorität des realen Lebens und des Vitalismus und besonders sein Begriff von Kunst als Lebenssteigerung, ja als größtes Stimulans des Lebens und als einzige "überlegene Gegenkraft gegen allen Willen zur Verneinung des Lebens, als das Anti-Christliche, Anti-Buddhistische, Anti-Nihilistische par excellence "12 für uns Heutige existentiell verbindlich sind - wie für die Expressionisten seinerzeit?! -

Anders gesagt: Gilt die ideologische Verurteilung Nietzsches von Georg Lukács bis S. F. Oduev oder haben Heinrich $\mathrm{Mann}^{13}$ und Albert Camus (L'Homme revolté, 1951) Nietzsche sozusagen richtig gelesen und für die „Moderne“ gedeutet und für unsere Zeit aus den fatalen Umklammerungen durch die NS-Faschisten befreit?

Damit hängt unmittelbar die obige Frage nach einer Differenzierung in die panegyrische und die emanzipatorische Nietzsche-Rezeption zusammen. Zwar wurde mir zu Recht entgegnet, daß die feiernde Nietzsche-Rezeption in konservativen und deutsch-nationalen Kreisen (z. B. Peter Behrens Vorhalle, Turin 1902) bis 1914 dominierte und die emanzipatorische Rezeption gering war. Aber gerade das veranlaßte mich, nach dieser zu fragen, da nur sie - im Sinne des Problems, ob Nietzsches Existenzphilosophie für uns genuin von Bedeutung sein kann (oder gar eine Synthese aus Marx und Nietzsche vorstellbar ist) ${ }^{14}$ - die emanzipatorischen Autoren (H. Mann, Franz Pfemfert, A. Camus) und Künstler (wie Bruno Taut, O. Dix) in eine Perspektive von Zukunft rücken kann. Nochmals anders gesagt: Nicht ein

seine Kunst, Köln 1976, 144-146. Besonders die wirren „100 Aphorismen - das zweite Gesicht“ von 1914 belegen deutlich den „Nihilismus“ Marcs im Sinne Nietzsches, d. h. seine Verleugnung der Priorität des Lebens und dessen obersten Wert, seine Abwertung des realen Seins. Dagegen Nietzsches „Geschichte eines Irrtums“ in Götzendämmerung (1888): „... mit der ,wahren“ Welt haben wir auch die scheinbare abgeschafft!“; vgl. dazu K. Löwith, Gott, Mensch und Welt, Göttingen 1967, $156 \mathrm{f}$.

12 „Die Kunst und nichts als die Kunst! Sie ist die große Ermöglicherin des Lebens, die große Verführerin zum Leben, das große Stimulans des Lebens. Die Kunst als die einzige überlegene Gegenkraft gegen allen Willen zur Verneinung des Lebens, als das Antichristliche, Antibuddhistische, Antinihilistische par excellence - Die Kunst als die Erlösung des Erkennenden ... / Die Kunst als die Erlösung des Handelnden ... / Die Kunst als die Erlösung des Leidenden ..." Dieser Text war auch 1908 in der Nachlaß-Texteauswahl des sog. Willens zur Macht, hg. v. P. Gast u. E. Förster, No. 853; F. Würzbach (Hg.), Umwerthung aller Werte, München 1977, 657 f. (KGW VIII/3, 319). Vgl. oben, Anm. 2 und P. Pütz, „Kunst und Künstlerexistenz im Werk Nietzsches“, in: Kunst und Künstlerexistenz bei Nietzsche und Thomas Mann, Bonn 1963, 15 f. („Die Kunst als Funktion des Lebens“!); D. Jähnig, Welt-Geschichte $=$ Kunst-Geschichte, Köln 1975.

13 H. Mann, Les pages immortelles de Nietzsche, Paris 1939, auch in: Mass und Wert, hg. v. T. Mann, 2. Jg., H. 3, 277-304; H. Mann, „Geist und Tat“ [1910], in: Mann-Essays, Hamburg 1960, 7-20. A. Camus, Nihilismus, 55 f. Zur Frage der Befreiung Nietzsches aus den Umklammerungen des NSFaschismus s. W. Kaufmann, Nietzsche - Philosopher, Psychologist, Antichrist, 4. Aufl., Princeton 1974, dt. v. J. Salaquarda, Darmstadt 1982.

14 Für manche Sozialisten um 1918 schlossen sich Marx als Sozialtheoretiker und Nietzsche als Existenztheoretiker offenbar nicht aus; eher ging es ihnen um eine Synthese: Jeder deckte einen Teil der Wirklichkeit ab bzw. erhellte ihn, - den sozialen Bereich und das Individuelle. Aber im Grunde ist Nietzsches lebensphilosophisches System letztlich eines für Artisten, für Künstler. - Georg Simmel löste den Widerspruch zwischen Nietzsche und Marx bereits 1907 auf (Schopenhauer und Nietzsche, Leipzig 1907, 207 f.). Vgl. ferner auch H. Grimm u. J. Hermand (Hg.), Karl Marx und Fr. Nietzsche, Königstein 1978; M. Montinari, „Nietzsche zwischen Alfred Baeumler und Georg Lukács“, in: Basis, H. 9 (1979), 194 f.; C. Türcke, Der tolle Mensch. Nietzsche und der Wahnsinn der Vernunft, Frankfurt a.M. $1989,105 \mathrm{f}$. 
Streiten um die Gewichtung solcher Nietzsche-Impulse scheint mir angebracht, eher die klare Herausstellung und dialektische Zusammenschau der kontrastierenden Impulse und Rezeptionen (z. B. Pfemfert contra „Deutschsprechung“ Nietzsches 1915); und darüber hinaus ist $\mathrm{zu}$ fragen:

Wie kann ein Impuls überhaupt adäquat beurteilt werden, wenn nicht die genaue Kenntnis der Philosophie Nietzsches vorangeht? Wie kann eine Wirkung auf Künstler differenziert beurteilt werden, wenn nicht eine exakte und deutliche Kenntnis von Nietzsches Begriff und Funktion der Künste/Kunst im Sinne seines Vitalismus und der Emanzipation und des Wachsens des Individuums („höherer Mensch“) vorangeht? Nur auf einem genauen Begriff von Nietzsches Rollenzuweisung an Kunst und ihrer lebensphilosophischen Funktion als „Stimulans des Lebens" und „Lebenssteigerung“ - für den einzelnen (Leidenden - Erkennenden - Handelnden), für das schöpferische Subjekt, also einer Künstler-Ästhetik, nicht einer für Zuschauer wie Kunsthistoriker - nur auf einer solchen Basis kann m. E. heute noch operiert werden.

Ohne diese Basis führt womöglich das Behandeln von Nietzsche-Wirkungsfragen zu Rezeptionsgeschichten mit Eigendynamik und ohne denjenigen Boden, aus welchem die kulturellen Gebilde wuchsen. Wie kann man Verfälschungen Nietzsches oder relevante Rezeption dann beurteilen?

Es wird Nietzsches Kunstbegriff, der der höchste bzw. tiefste und gewichtigste in der "Moderne" seit Heine und Baudelaire ist (gerade in der Kritik an Kants und Schopenhauers Schönheits- und Kunstbegriff), ${ }^{15}$ ebenso erhellt, wenn er einen Komponisten wie Beethoven oder Wagner, einen Dichter wie Stendhal oder einen Maler wie Delacroix als ExistenzTypus seines Horizontes charakterisiert. Deshalb auch hier die Frage nach den DelacroixTexten bei Nietzsche als Mosaiksteine.

„Wie Schopenhauer nur einen einzigen absoluten Wert kennt: Nicht-Leben, - so kennt Nietzsche gleichfalls nur einen: Leben. Wie für jenen alle sonst als selbständig anerkannten Werte: Schönheit und Heiligung, metaphysische Vertiefung und Sittlichkeit nur Mittel sind, die auf das Endziel der Verneinung des Lebens ausgehen, so sind diese und alle anderen Güter und Vollkommenheiten für Nietzsche ein Mittel der Bejahung und Steigerung des Lebens. "16

15 Besonders in der Analyse der asketischen Ideale Schopenhauers hat Nietzsche die Nachwirkung von Kants „interesselosem Wohlgefallen“ konstatiert und dem Stendhals Begriff der Wirkung des Schönen (Natur- und Kunstschönen) als eine „promesse de bonheur“ - „willenerregend!“ - entgegengesetzt (GM [1887], KGW VI/2, 3. Abh., No. 6). Ich habe dies 1983 hinsichtlich der Beckmann-Marc-Kontroverse von 1912 im Band Expressionismus und Kulturkrise, hg. v. B. Hüppauf, Heidelberg 1983, 228, schon einmal thematisiert. Besonders die Aufsätze von Volker Gerhardt beleuchten die Bedeutung der Rolle der Kunst in Nietzsches vitalistischem System: „Artisten-Metaphysik“, in: Zur Aktualität Nietzsches, Bd. I, Würzburg 1984, 81-91; V. Gerhardt, „Von der ästhetischen Metaphysik zur Physiologie der Kunst“, in: Nietzsche-Studien 13 (1984), 374 f.; V. Gerhardt, Friedrich Nietzsche, München 1992; ferner H. Pfotenhauer, Die Kunst als Physiologie. Nietzsches ästhetische Theorie, Stuttgart 1985, 202 (Nietzsche als Leser Baudelaires). Zu Heinrich Heine als Autor der „modernité“, der Baudelaire beeinflußte, H. R. Jauss, Literaturgeschichte als Provokation, Frankfurt a.M. 1970, 53, 107, 140; B. Steinwachs, ,,Zeitbewegung" als Signatur der Moderne“, in: Epochenbewußtsein und Kunsterfahrung, München 1986, 259.

16 G. Simmel, Schopenhauer und Nietzsche, Leipzig 1907, 195. 
Dieses Fazit schrieb Georg Simmel 1907 in seinem Schopenhauer-Nietzsche-Buch am Beginn des Kap. 7, Die Menschheitswerte und die Dekadenz. Vor diesem Hintergrund ist es evident, daß der Kunst-Begriff Nietzsches ein gänzlich anderer sein $m u \beta$ als der Schopenhauers. Auch müssen bei beiden Denkern andere Künstler in den Blick geraten und entsprechende Signifikanz besitzen.

Damit komme ich zu Nietzsches Blick auf Eugène Delacroix und seiner Auslegung dieses bedeutendsten Malers und Zeichners des 19. Jahrhunderts als schöpferischen Subjekts (Abb. 1, Selbstporträt, Louvre).

Vorauszuschicken ist, daß Nietzsche sich sonst mehr mit Musikern wie Wagner, Beethoven und Brahms auseinandersetzte, weniger mit Malern der jüngeren Historie. Es ist bekannt, welche Rolle Raffaels Transfiguration (im Vatikan-Museum) für Nietzsches Frühschrift und die Verdeutlichung von „dionysisch“ und „apollinisch“ spielte. Auch wurde früh immer das besprochen, was Nietzsche aus der Kunstgeschichte kannte und lobte - seit Georg Fuchs 1895 bis Theo Meyer 1993. ${ }^{17}$

Dabei stand er teils unter Jakob Burckhardts Normen, so daß ihm wegen dessen verständnisloser Negation der überragende Rembrandt regelrecht imprägniert war. ${ }^{18}$

Nennt Nietzsche in den Spätschriften für seine Perspektiven bedeutende Persönlichkeiten (in JGB 1886 oder in GD 1888), etwa die für die europäische Kultur mitzählenden, so fallen die Namen Beethoven, Goethe, Wagner, Schopenhauer, Heinrich Heine, Stendhal.

Den bildenden Künstler Auguste Rodin, dessen vitalistische Werke durch Simmel als "moderner Heraklitismus" definiert wurde, ${ }^{19}$ kannte Nietzsche nicht. Möglicherweise hätte diese Persönlichkeit Nietzsches Vorstellungen auch exemplifizieren können, - wie die Person und der Stil von Delacroix.

Im Aphorismus 256 in Jenseits von Gut und Böse 1886 beschreibt Nietzsche die Verwandtschaft der europäischen Spätromantiker unter dem Impuls des Suchens einer neuen, ungestümen Kunst, - die „hinaufdrängt ... wohin? in ein neues Licht?“"20

17 G. Fuchs, „Nietzsche und die bildende Kunst“, in: Kunst für Alle 11 (1895/96), 33 f., 71 f., 85 f. Wilhelm Stein, „Nietzsche und die bildende Kunst", in: Archiv für Geschichte der Philosophie und Soziologie 30 (1925), H. 1/2; T. Meyer, Nietzsche und die Kunst, Tübingen/Basel 1993, 110 kurz zu Delacroix (aber ohne auf Aphorismus 256, Jenseits von Gut und Böse einzugehen!).

18 Das Fehlurteil J. Burckhardts über Rembrandt ist nach wie vor unbegreiflich und hat im späten 19. Jahrhundert reichlich Verwirrung gestiftet; offenbar war Burckhardt im Grunde Klassizist. Daß Rembrandt nicht zeichnen könne, ist eins der größten Fehlurteile der Kunsthistorie, ebenso begriff Burckhardt nicht, daß Rembrandt durch und durch Kolorist war. Und daß das Licht nicht Eigenzweck, sondern schließlich Rembrandts Mittel war, die Menschengestalt seelisch tiefer zu veranschaulichen, verstand Burckhardt nicht. (Man vergleiche dagegen die tiefe Deutung Rembrandts bei G. Simmel, Rembrandt, Leipzig 1916.) Nietzsche hat wegen Burckhardts Wirkung Rembrandt für sich nicht entdecken können. J. Burckhardt, „Rembrandt“ (1877), in: Die Kunst der Betrachtung. Aufsätze und Vorträge, Köln 1985, 384 ff.

19 G. Simmel, „Rembrandt“, 134-137; der früheste Beitrag Simmels zu Rodin erschien im Berliner Tageblatt vom 29. Sept. 1902, vgl. dazu D. Schubert, Die Kunst Lehmbrucks, 2. verb. Aufl. 1990, 49; J. A. Schmoll-Eisenwerth, „Simmel und Rodin“, in: Rodin-Studien, München 1984. Zum Heraklitismus bei Nietzsche vgl. vor allem W. Gebhard, Nietzsches Totalismus - Philosophie der Natur zwischen Verklärung und Verhängnis, Berlin/New York 1983, $70 \mathrm{f}$.

20 JGB, „Völker und Vaterländer“, 1886, No. 256, KGW VI/2, $210 \mathrm{f}$. 


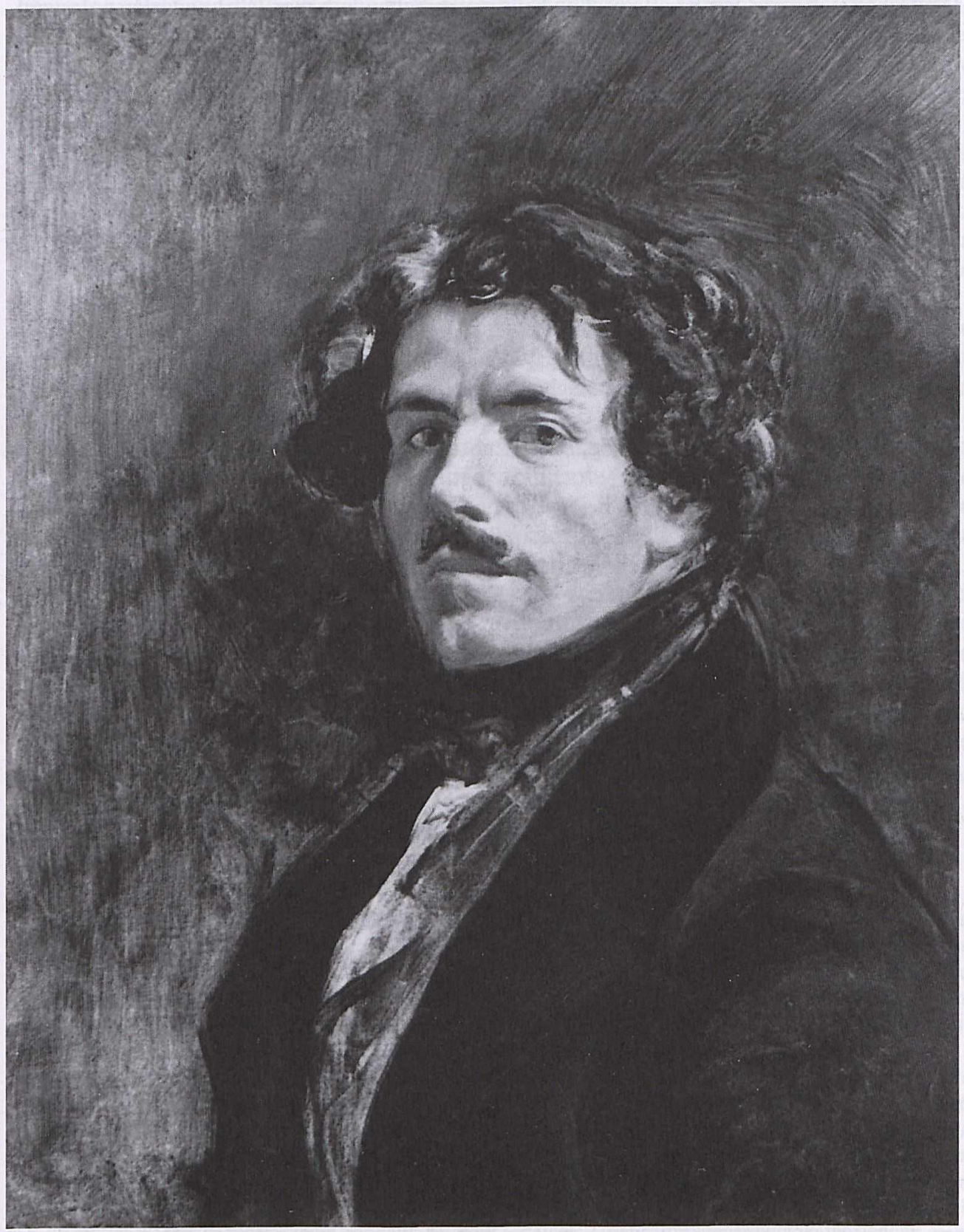

Eugène Delacroix: Selbstporträt, um 1837, Louvre, Paris 
Der Kontext Nietzsches ist die Idee Europa, „daß Europa eins werden will“. Dies sei der Wille und das Ziel aller tieferen Menschen des 19. Jahrhunderts, - „versuchsweise den Europäer der Zukunft vorwegzunehmen“. Die Ablehnung der Krankheit des Nationalismus, der névrose nationale, die Nietzsche vor allem dem Deutschen Reich und dem Typ des Deutschen vorwarf (dem er alle großen Kulturverbrechen von vier Jahrhunderten anlastete), ist Ausgangspunkt von No. 256 in JGB: Krankhafte Entfremdung in Europa sei die Folge des „Nationalitäts-Wahnsinns“, gegen den nur die „umfänglicheren Menschen“ stünden. Nietzsche nannte hier 1886 nochmals bzw. wieder Richard Wagner. Aber das Urteil über diesen radikalisierte sich negativ bis 1888 , als er Deutschtümelei, Antisemitismus und Siegfried-Rassismus bei Wagner deutlicher spürte und in den Texten Der Fall Wagner 1888 und im Ecce homo (Der Fall Wagner, Abs. 2) und in Nietzsche contra Wagner 1888 präzise und scharf darstellte. ${ }^{21}$

Jahre zuvor beließ er Wagner auch im Kontext des Künstler-Typus, den er im Lichte der „Synthesis“, daß „Europa eins werden will“, charakterisiert, den europäischen Spät-Romantiker, Meister des Sturm und Drang,

„diese letzten großen Suchenden! [...] allesamt Fanatiker des Ausdrucks - à tout prix ich hebe Delacroix hervor (den Nächstverwandten Wagners) - allesamt große Entdecker im Reiche des Erhabenen, auch des Häßlichen und Gräßlichen, noch größere Entdecker im Effekte, in der Schaustellung, ... Virtuosen durch und durch, mit unheimlichen $\mathrm{Zu}$ gängen zu allem, was verführt, lockt, zwingt, umwirft, geborene Feinde der Logik“

- und nun eine Metapher, die m. E. besonders signifikant ist auch für die Pole innerhalb der späteren Moderne zwischen Beckmann/Picasso einerseits und Mondrian/Malewitsch andererseits - und

Feinde „der geraden Linien, begehrlich nach dem Fremden, dem Exotischen, dem Ungeheuren ... dem Sich-Widersprechenden; als Menschen Tantalusse des Willens, heraufgekommene Plebejer ... man denke z. B. an Balzac -, zügellose Arbeiter, beinahe SelbstZerstörer durch Arbeit; Antinomisten und Aufrührer in den Sitten, Ehrgeizige und Unersättliche ohne Gleichgewicht ..., im Ganzen eine verwegen-wagende, prachtvoll-gewaltsame, hochfliegende und emporreißende Art höherer Menschen, welche ihrem Jahrhundert - und es ist das Jahrhundert der Menge! - den Begriff höherer Mensch erst zu lehren hatte."

21 In Nietzsche contra Wagner („Wie ich von Wagner loskam“) 1888 schreibt er, daß er sich bereits 1876 innerlich trennte: „seitdem Wagner in Deutschland war, kondeszendierte er Schritt für Schritt zu allem, was ich verachte - selbst zum Antisemitismus"! Vgl. auch schon Fröhliche Wissenschaft 1882, No. 99. Der im folgenden referierte Text über Wagner und Delacroix stimmt in wesentlichen Teilen überein mit „Wohin Wagner gehört" in Nietzsche contra Wagner; er nahm also die Aussagen von 1886 in diese spätere Schrift auf, nun aber ohne Delacroix zu nennen. Im entsprechenden Text in Ecce homo 1888 endet er aber mit dem Begriff „Künstler“ anstelle „höherer Menschen“. - Wieder taucht der Name Delacroix im gleichen Kontext der französischen Spätromantik auf, nun zusammen mit Baudelaire und Berlioz als drei „Fanatiker des Ausdrucks“ 1888 im Ecce homo („Warum ich so klug bin“, Abs. 5), wobei der Schlußgedanke wiederum lautet: „Was ich Wagner nicht vergeben habe? daß er zu den Deutschen kondeszendierte, - daß er reichsdeutsch wurde ..." 
Delacroix - einer der höheren Menschen?

Bevor Fragen an diesen Text herangetragen werden, noch eine Zwischenbemerkung zur Idee des „höheren Menschen“: Dieser Ausdruck ist - ebenso wie die Idee vom Tod Gottes - von Jean Paul entlehnt bzw. weitergeführt, den Nietzsche bekanntlich las und als „Verhängnis im Schlafrock“ beschrieb. Auch Nietzsches Ausdruck „Übermensch“ - der im Delacroix-Text nicht fiel - den er einmal definierte als "halb Heiliger, halb Genie“, begegnet bereits bei Jean Paul - ähnlich "Genie“, „Hochmensch“, und zwar in einem Kontext wie bei Nietzsche, nämlich bezogen auf Napoleon Bonaparte: - „das verwüstende Feuer solcher Übermenschen" - in Jean Pauls Essay über Charlotte Corday 1801.

Für eine Auslegung von Nietzsches Passage über Delacroix scheinen mir zwei Fragen angebracht: 1. Wie kommt Nietzsche zu diesem überaus treffenden Urteil? - Welche sind seine Quellen? Literatur oder Anschauung? Welche Werke des Malers kannte er? 2. Deckt sich seine Charakteristik mit unserer Sicht des vitalistischen Malers/Zeichners und Führers einer modernen "neuen Malerschule" in Frankreich, - wie ihn schon Heinrich Heine im Salonbericht 1831 und im Nachtrag 1833 begrüßt hatte?22

Es kann nicht gesagt werden, welche Gemälde Delacroix' Nietzsche im Original kannte (aus Basel, Zürich, Genua, Turin, Paris?). Aus welchen Büchern er Kenntnis von Delacroix schöpfte, läßt sich nach meinen Recherchen annähernd sagen: Baudelaires Salontexte 1845/46 kannte er (zum Teil aus Zitaten im Delacroix-Buch von G. Dargenty, Paris 1885) und insbesondere, was die weiter unten noch heranzuziehenden zwei Nachlaß-Texte, in denen Delacroix figuriert, betrifft: Edmond und Jules de Goncourts Künstlerroman Manette Salomon von 1867 (2. Ed. 1876), aus dem er nachweislich exzerpierte, der jedoch nicht in seiner Bibliothek in Weimar erhalten ist. ${ }^{23}$ Das hieße, daß Nietzsches Begriff von Delacroix primär aus zweiter Hand stammte, wenn er keine Originale sah.

Zur zweiten Frage: Die Charakteristik Delacroix' stimmt durchaus mit unserer modernen Sicht überein: Delacroix der bedeutende Kolorist (Aufbau des Bildes aus der Bewegung der Farbe, nicht aus der Konturzeichnung; Negation der Einzellinie, Zeichnen in Ovalen und in Schraffuren; - das Werk aufbauen nicht "par la ligne mais par les milieux“ (aus den Mitten), sein Primat der Leidenschaft (émotion et passion) und Lebendigkeit (vivacité) und der künstlerischen expression; das Interesse für das Exotische (Nordafrika); vor allem die Kontra-Stellung zur David-Schule und zu Ingres als dem Haupt der Linearisten/Klassizisten;

22 H. Heines Delacroix-Text in Gemäldeausstellung 1831, die Bewertung von Géricault insbes. als dem Eröffner der neuen Malerschule in Frankreich im Nachtrag 1833 (K. Briegleb, Heine. Sämtliche Schriften, Bd. III, 1975, 39 f., 76). Dazu W. Hofmann, „Heine und die Malerei der Zukunft“, in: Heine-Jahrbuch, Düsseldorf 1981, 72 f.; K. Briegleb, Opfer Heine? Versuche über Schriftzüge der Revolution, Kap. 5: „Melancholischer Realismus. Heines Weg an die Seite Baudelaires“, Frankfurt a.M. 1985, 125 f.; B. Steinwachs, „Zeitbewegung“, 257 f.

23 Im August 1995 habe ich in Nietzsches Bibliothek nach Literatur über Delacroix gesucht: Baudelaires kunstkritische Schriften sind nicht vorhanden; Manette Salomon von den Brüdern Goncourt hat sich nicht erhalten (verschenkt?); noch vorhanden mit Nietzsches Anstreichungen ist G. Dargenty, Delacroix par lui mème, Paris 1885, wo Nietzsche (bes. 210-212) Zitate aus Baudelaires Texten über Delacroix zum dessin, zu le surnaturalisme u. a. hervorhob. Unvollständig ist also, was den ursprünglichen Bestand betrifft, das Heft von M. Oehler, Nietzsches Bibliothek, 13. Jahresgabe d. Ges. d. Freunde des Nietzsche-Archivs, Weimar 1942. Auch Stendhals De l'amour von 1822, das Nietzsche kannte, ist nicht erhalten. 
Delacroix ein Dionysiker, der als Ziel hat „frapper l'imagination“. Das Bild muß von uns Heutigen nicht revidiert werden. ${ }^{24}$

Um Nietzsches Charakterisierung näher zu begreifen, hebe ich nochmals hervor: „Fanatiker des Ausdrucks“, ${ }^{25}$ - große Entdecker im Effekte, mit unheimlichen Zugängen zu allem, was verführt, zwingt; geborene Feinde der Logik und der geraden Linien; zügellose Arbeiter, Menschen des Willens, begehrlich nach dem Exotischen, Aufrührer, Unersättliche.

Es kann hier nicht jeder Begriff und Ausdruck Nietzsches auf Delacroix' Kunst hin reflektiert werden. Aber ich greife noch die Metapher der Linie heraus, um auf die damals revolutionäre Zeichnungstechnik von Delacroix hinzuweisen. Tatsächlich trifft Nietzsches Formulierung den Kern von Delacroix' Anti-Klassizismus (er warf der Ingres-Schule ihren Pedantismus vor), den der Philosoph unter „französische Spätromantik“ subsumiert. Delacroix lehnte nicht nur die klassizistische Linie als eine Abstraktion und als „kalt" ab, er betonte vielmehr, daß es in der Natur die Linie überhaupt nicht gibt und forderte einen expressiven Zeichenstil, der in variierten Doppel- und Dreifach-Konturen, in Ovalen und in Schraffuren den Raum, die Volumen und die Plastizität der Figuren und anderen Sujets erfaßt, - nicht im Detail, nicht aufbauend auf der einzelnen Kontur, sondern aus den Massen; Brüche und Auslassungen, Verdichtungen, insbesondere durch Verwendung der Tusche (um bewegte Massen zu geben), Dunkel gegen Hell - das waren Elemente seiner eminent modernen Zeichnungstechnik (die $\mathrm{z}$. T. von Rubens und Rembrandt inspiriert war).

Im Journal lesen wir am 11. Januar 1857 unter den Stichworten für sein Dictionnaire des Beaux-Arts: „Dessin, - par les milieux ou par le contour.“

Der jüngere Künstler Jean Gigoux hat 1885 in seinen Causeries die Explizierung von Delacroix hinsichtlich der Polarität des Zeichnens - Kontur (Ingres) oder in Massen (Delacroix) - überliefert; Delacroix sagte: „Les antiques prenaient par les milieux, au lieu que la Renaissance prenait par la ligne“, ${ }^{26}$ - ein Programm, das Charles Blanc in seine Gram-

24 J. Meier-Graefe hat das große Verdienst, im wilhelminischen Deutschen Reich die Bedeutung Delacroix' bewußt gemacht zu haben; die Künstler wie van Gogh, Cézanne, Signac hatten dies längst durch Originale und durch die Schriften von C. Blanc, „Delacroix“, Gazette des Beaux-Arts 16 (Jan. - Febr. 1864), 5-27, 97 f.; C. Blanc, Grammaire des Arts du dessin, Paris 1867, 4. Aufl. 1881 (die auch van Gogh studierte). Die Polarität von Linearismus (Tradition Holbein) und Kolorismus (Tradition Tizian, Velazquez) findet sich bereits in Balzacs Novelle Le chef-d'euvre inconnu von 1831, wo Holbein und Tizian als die Leitbilder genannt sind; - aber auch bei Baudelaire und bei T. ThoréBürger; später hat sie klar J. Meier-Graefe operationalisiert, auch zu seiner Verwerfung von Arnold Böcklin 1905 (Der Fall Böcklin und die Lehre von den Einheiten, Stuttgart 1905), die die Geister schied - gestern wie heute. J. Meier-Graefe (Hg.), Delacroix. Literarische Werke, Berlin 1910; K. Schawelka, Delacroix. Sieben Studien zur Kunsttheorie, Mittenwald 1979, 112 f.

25 Nietzsche verwendete den Begriff „Ausdruck“/,Fanatiker des Ausdrucks“ auch in Die Unschuld des Werdens, in: Jenseits von Gut und Böse, No. 256 und im entsprechenden Text im Ecce homo („Warum ich so klug bin“, No. 5); vgl. dazu B. Hillebrand, Artistik und Auftrag, 170.

26 C. Blanc, Grammaire des Arts; van Gogh, Sämtliche Briefe, hg. v. F. Erpel, Bd. 3, Berlin 1965, 248 f. Zu Delacroix’ Zeichnungsstil vgl. K. Badt, Eugène Delacroix - Drei Abhandlungen, Köln 1965, 9-45. (auch sep. publ. Baden-Baden 1951). Der Louvre zeigte 1982 eine umfassende Schau der dortigen Blätter Delacroix' - ohne Katalog: Revoir Delacroix, 19.6.-22.9.1982; vgl. auch K. Schawelka, Delacroix 1979, der der Zeichnung jedoch kein Kapitel widmete. M. Sérullaz, Dessins d'Eugène Delacroix 1798-1863, Bd. 1-2, Paris 1984. Auf der Basis der Tagebücher Delacroix' hat Badt seinen Zeichnungsstil gültig erläutert, die Elemente habe ich oben kurz genannt. Gute Beispiele sind die Studien zum Sardanapal und zur Medea das Blatt im Musée Bonnat in Bayonne (V. Ducourau, Catalo- 
maire 1867 aufnahm, aus der es 1885 Vincent van Gogh im Brief 401 an seinen Bruder wiedergibt. Ein Blick insbesondere auf die Vorstudien um 1836 zur Medea in den Museen Lille (Abb. 2) und Bayonne kann dies veranschaulichen.

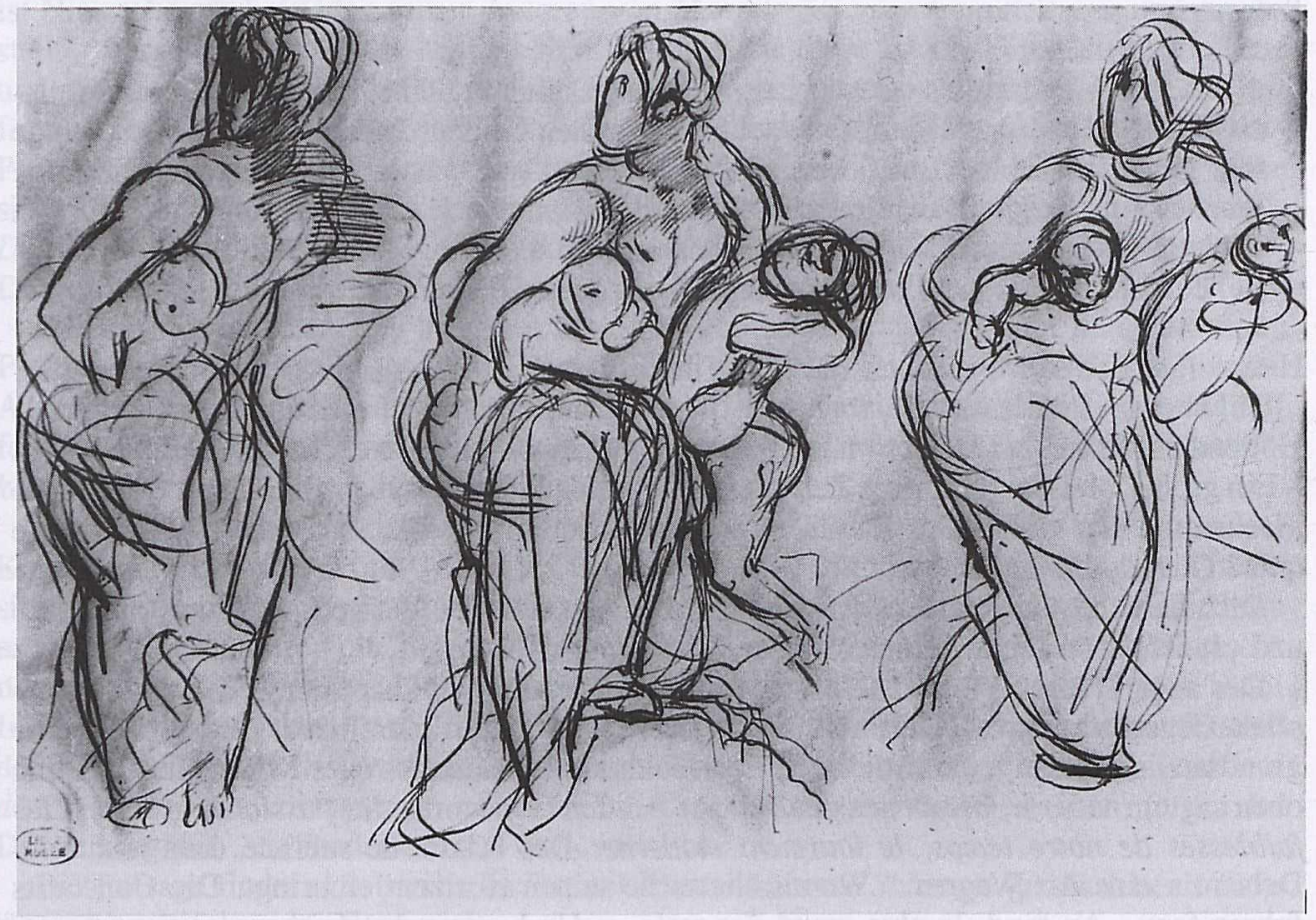

Eugène Delacroix: Studie zur Medea, um 1836 (Lille)

Ganz zutreffend ist auch Nietzsches knapper Begriff „Fanatiker des Ausdrucks“. Delacroix betonte häufig als Basis seines Kunstwollens die Begriffe der „vivacite“, „expression“ und „exagération“, besonders im Tagebuch von 1857, wo er Termini für das geplante Dictionnaire des Beaux-Arts auflistete. ${ }^{27}$ Auch Delacroix Stichworte "surnaturel" und "le terrible" (25.1.1857) koinzidieren mit Nietzsches Vorstellungen wie das Ungeheure oder pessimistisch tragische Mentalität.

Drei Nachlaßtexte aus 1884, von Colli/Montinari 25 (141-144) numeriert, können gewissermaßen als die Ausgangsbasis von Nietzsches Delacroix-Bild in seinen Buchtexten verstanden werden; die Quelle seiner Texte, die im Grunde nur Exzerpte waren, gibt er selbst

gue Dessins français du XIXe siècle du Musée Bonnat, Paris: Louvre 1979, Nr. 49 u. 53). Zu den Ovalen in Delacroix' Theorie vgl. T. Silvestre, Eugène Delacroix. Documents nouveaux, Paris 1864 , 2. Aufl. 1926, 42; J. Gigoux, Causeries sur les artistes de mon temps, Paris 1885; K. Badt, Eugène Delacroix, 38 f. Baudelaire hatte bereits 1846 die Polarität zwischen Ingres und Delacroix gespürt auch die Kontraste betreffs Zeichnung und Kolorismus (C. Baudelaire, Werke, Bd. 1, hg. v. F. Kemp, 1977, 214). Eventuell ist Nietzsche durch die Lektüre Heines und Baudelaires überhaupt erst auf Delacroix' Kunst aufmerksam geworden und las dann Goncourts Manette Salomon?

27 E. Delacroix, Journal 1822-1863 (edition Plon), von A. Joubin, Paris 1982, 612-623. 
an: „M. Salomon I, p. 197“, also den Künstlerroman der Brüder de Goncourt. Als die Basis und Quelle für die Delacroix-Charakterisierung in Jenseits von Gut und Böse gebe ich sie hier weitgehend wieder und vergleiche diese Exzerpte mit dem Originaltext in Manette Salomon.

„Delacroix - er hat alles versprochen, Alles angekündigt. Seine Bilder? foetus von Meisterwerken; der Mensch, der, après tout, am meisten Leidenschaften erregen wird comme tout grand incomplet. Ein fieberhaftes Leben in allem, was er schafft, une agitation de lunettes [im Original hier jedoch ,agitation de tumulte'], un dessin fou - er sucht la boulette du sculpteurs, le modelage de triangles (et de losanges im Vorbild) qui n'est plus (le) contour de la ligne d'un corps, mais l'expression, l'épaisseur du relief de sa forme."

Hier folgte bei den Goncourts die Frage nach dem Koloristen; Nietzsche notiert weiter: „[Un] harmoniste désaccordé, tragische Unterfarben [im Original ,des tonalités tragiques'], Höllendämpfe wie bei Dante. “ Nietzsche übergeht mehrere Zeilen; „Es giebt keine Sonne. - Ein großer Meister für unsere Zeit aber, im Grunde la lie de Rubens. “ Bei den Goncourts: „Delacroix! Un grand maître? oui, pour notre temps [...] Mais au fond, ce grand maître quoi? C'est la lie de Rubens! “”

Dichtheit und Ausdruck exzerpierte Nietzsche gesperrt. Die Verwechslung von "tumulte“ und „lunette“ dürfte ein Lesefehler der Editeure sein?28

Der andere Text (NF II, 25/141, 51), in dem Nietzsche in knappster Weise den historischen Gegensatz Ingres - Delacroix, der keine Frage von „Stilpluralismus“ ist, sondern eine grundsätzliche geistige, künstlerische Entscheidung: Linearismus oder Kolorismus (wie ich oben sagte), notierte, betont aus der Passage bei den Goncourts „les passions, les nerfs, les faiblesses de notre temps, le tourment moderne. Des éclairs de sublime dans tout cela. Delacroix eine Art Wagner." Womit Nietzsche seinen Horizont einbringt. Die Goncourts fuhren fort: „Au fond, le plus grand des ratés ... Un homme de génie, venu avant terme. Il a tout promis ..." Hier schließt Nietzsches Notiz 15 (142) an.

$\mathrm{Da}$ der David-Nachfolger und an Perugino und Raffael orientierte Klassizist Ingres, dessen Malstil eine zeichnerisch genaue, gleichmäßig beleuchtete Wiedergabe aller Gegenstände im Bilde ohne "Opfer" (wie Delacroix sie im Anschluß an Rembrandt forderte), seinen Kontrahenten Delacroix einmal als „l'apótre du laid“ (des Häßlichen) beschimpfte, mag als Pointe hier zitiert sein. ${ }^{29}$

Dagegen verstand als erster nach Heine und T. Gautier der Dichter Baudelaire 1845/46 in seinen Salontexten die Zeichnungsweise und den tieferen Gehalt der Malerei von Delacroix. Nietzsche kannte diese aus der Lektüre von Dargenty, und das geht ferner aus den Notizen von 1885 für Jenseits von Gut und Böse, No. 256 hervor: „Delacroix übrigens auch sehr Musiker ... sein erster Interpret Baudelaire, eine Art R W ohne Musik. Der Ausdruck expression von Beiden vorangestellt, alles übrige geopfert. Von Litteratur abhängig

28 Dies ist nur eine Vermutung und wäre am Original zu prüfen. - Daß es bei Delacroix keine „Sonne“ gäbe, was Nietzsche mehrmals von den Goncourts übernimmt, ist freilich eine Vereinfachung der Franzosen; - es scheint uns heute nicht derartig zu sein: Die erotischen Frauenbilder, manche Landschaft bei Valmont und Dieppe/Etretat widersprechen solcher Gesamtwertung.

29 J. D. Ingres hier zit. nach K. Schawelka, Delacroix, 48. Manche Kenntnis von neuer Malereigeschichte hatte Nietzsche auch aus H. Taine, Philosophie der Kunst, dt. Ed. 1866 (in seiner Bibliothek Nr. 498). 


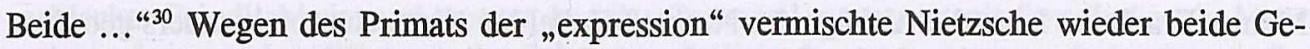
stalten, Wagner und Delacroix, als quasi einen Typus.

Zur historischen Opposition der zwei gegensätzlichen Malerschulen Delacroix - Ingres ist Nietzsche am Leitfaden seiner Autoren von Manette Salomon unmittelbarer und unverstellter vorgedrungen als die heutige Kunsthistorie, die diese kontrastierenden Phänomene und grundsätzlichen Entscheidungen als „Stilpluralismus“ harmonisieren möchte. Er zitiert für sich aus dem Roman der Goncourts die entscheidende Einschätzung der beiden MalerProtagonisten als Pole in der Malerei des 19. Jahrhunderts: „Ingres: 1'inventeur au 19me siècle de la photographie en couleur pour la reproduction des Pérugin et des Raphael. Delacroix c'est 1'antipôle ... “ Bei den Goncourts lesen wir nach Raphael „voilà tout! ... Delacroix, lui, c'est l'autre pôle - Un autre homme".

Und im Nachlaßtext 25/144 exzerpierte sich Nietzsche nochmals eine komplementäre Passage, die die Klassizisten und Salonmaler Ary Scheffer und Delaroche als „TheaterArrangeure" sieht - mit "täuschenden Lokalfarben“" danach mit der entscheidenden Schlußfolgerung, die zutrifft: „aber das Leben fehlt. - Solche Maler im Grunde sterile Persönlichkeiten."

Den Schluß seiner Notizen zu jenem Komplex der Malereigeschichte bildet also der Kontrast Ingres - Delacroix, - als die "beiden extremen Naturen“. In der Lage schlossen sich die Jungen überwiegend Ingres Stil an, die wenigeren jedoch Delacroix, der „le beau expressif“" verkörpere, die Ingres-Schule jedoch „le Beau de Raphael“. Und Nietzsche notierte sich etwas von großer Tragweite für die Kunstgeschichte der „Moderne“" nach 1855: In jener Ermüdungslage des Klassizismus, Historismus und der Salonkunst zeige niemand den jungen Talenten das, was am meisten verachtet werde: die eigene Zeit, „ce grand côté dédaigné de l'art: la contemporanéité ". ${ }^{31} \mathrm{Die}$ Goncourts verbanden diese Idee mit der der Darstellung der "vie moderne" und der "verite“".

Die Passage findet sich bereits im Kapitel III von Manette Salomon (geschrieben 1866); sie endet mit folgenden Sätzen:

„Mais ce n'était qu'une minorité, cette petite troupe de révolutionnaires qui s'attachaient et se vouaient à Delacroix, attirés par la révélation d'un Beau qu'on pourrait appeler le Beau expressif. La grande majorité de la jeunesse, embrassant la religion des traditions et voyant la voie sacrée sur la route de Rome, fêtait rue Montorgueil le retour de M. Ingres comme le retour du sauveur du Beau de Raphael. Et c'est ainsi qu'avenirs, vocations toute la jeune peinture, à ce moment, se tournaient vers ces deux hommes dont les deux noms étaient les deux cris de guerre de l'art: Ingres et Delacroix. “32

30 C. Baudelaire, Delacroix (Salon 1845 und 1846), in: Werke, 130 f., 206 f., „Delacroix - das Haupt der modernen Schule". (NF, KGW II, 476)

31 Der Nachlaßtext beginnt mit der Lage der Romantik um 1840, den „peintres poètes“, und kommt dann zu Scheffer und Delaroche - „Schüler Walter Scotts“ (NF, KGW II, 52); - Nietzsche schrieb aus Kapitel III des genannten Romans ab, in welchem die Goncourts die Lage des Romantisme ums Jahr 1840 charakterisierten. Siehe im folgenden.

32 E. u. J. de Goncourt, Manette Salomon, hg. v. L. Descaves, Paris o. J., 23; die obigen Zitate zu Ingres/Delacroix $151 \mathrm{f}$. Eine andere Ausgabe von H. Juin, Paris 1979 (Série Fins de siècles), $30 \mathrm{f}$., 142 f.; vgl. K. v. Maur, Französische Künstler des 19. Jh. in den Schriften der Brüder Goncourt (Diss.), Tübingen 1967. 
Meines Erachtens kann die Abfolge der Nachlaß-Zettel bei Nietzsche somit anders geordnet werden: Nr. 144 vor 141, da das Exzerpieren sicher der Lektüre folgte.

Damit hatte sich Nietzsche nicht nur den folgenreichen Kontrast Delacroix - Ingres klargemacht und in Details der Realisation von Linie/Zeichnung und Farben erkannt, mehr noch: Im Zentrum, im Fragekreis der "vivacite“, der Ermöglichung von Leben, stehen Frage und Appell als Eines: wirkt das Kunstwerk in höchster Lebendigkeit (wenn es sie verkörpert, wenn das Leben nicht fehlt)?

Nicht als sogenannter „Pluralismus“, wie heute von den Ästheten ständig abgeschwächt wird, ${ }^{33}$ nein, als Anti-Pole, als konkurrierende, sich ausschließende, ja verfeindete Positionen, konnte Nietzsche für seinen Kunstbegriff jene historische Lage verstehen; - eine Polarität, die blieb, die sich wiederholte und die sich heute in veränderten Ausdrucksformen wiederholt: um 1889 mit Gauguin und Vincent van Gogh, um 1912 deutlich mit Franz Marc und Max Beckmann, heute mit Abstrakten wie U. Rückriem und Donald Judd und expressiven Realisten wie Alfred Hrdlicka oder Georg Baselitz. Gerade die Verachtung der Kontemporaneität, die Nietzsche aus seiner Lektüre exzerpierte, $d . h$. die ihm signifikant erschien, ist eine Pointe, die Geltung hatte und behält: Die heutige Verachtung der expressiven Menschendarstellung und des expressiven Realismus, die von den Ideologen der Abstrakte als „konventionell“ abgetan werden, gleicht der Wut Calibans, als er sein eigenes Antlitz im Spiegel erblickte (O. Wilde 1890), ${ }^{34}$

In einem letzten Gedankengang möchte ich die vorgestellte Beziehung in das Licht einer für Nietzsches Kunstbegriff signifikanten Passage contra Schopenhauer in der Genealogie der Moral (1887) rücken. Unter der Frage „Was bedeuten asketische Ideale?“ analysierte Nietzsche die Feindschaft Schopenhauers gegen die Sinnlichkeit, das Weib, die Geschlechtlichkeit und zugleich dessen (falschen) Kunstbegriff. Von Kant ausgehend habe Schopenhauer einen Kunstbegriff ohne „Selbst-Erfahrung“, habe über „die Kunst und das Schöne“ allein vom Zuschauer aus nachgedacht, und er komme somit zu der Formel, das NaturSchöne und das Kunst-Schöne seien allgemein und unpersönlich und „was ohne Interesse gefällt". Nietzsche erkennt in dieser, für ihn falschen Vorstellung die Wurzel der Ge-

33 Die signifikante Stelle bei den Goncourts über „la guerre de l'art“ um 1855 ist nicht berücksichtigt bei G. F. Gerlach, „Ingres und Delacroix“, in: Ingres und Delacroix (Katalog d. Ausst. Tübingen/Brüssel), hg. v. E. Goldschmidt u. G. Adriani, Köln 1986, 17-23. Vgl. ferner jetzt hinsichtlich der „Schule von Barbizon“ J. Hauck, „Die Chimäre der Objektivität“, in: Barbizon (Katalog d. Ausst.), hg. v. C. Heilmann, M. Clarke u. J. Sillevis, München 1996, 62, 71, Anm. 16; Hauck bezeichnet Manette Salomon als einen der reichhaltigsten literarischen Texte zur Malerei des 19. Jahrhunderts.

34 Die Caliban-Metapher aus Shakespeare verwendet von Oscar Wilde, Vorwort zum Dorian Gray, 1890 (vgl. D. Schubert, Otto Dix, 136). Zur Beckmann-Marc-Kontroverse von 1912 vgl. Katalog Beckmann - die frühen Bilder, hg. v. U. Weisner, Bielefeld (KH) 1982; und meinen Beitrag in Expressionismus und Kulturkrise, 207-244. Der polare Kontrast zwischen Minimalisten wie Carl André und Donald Judd einerseits und Künstlern wie Georg Baselitz und Alfred Hrdlicka andererseits wird in der heutigen Kunsthistorie nicht thematisiert; entweder man spricht von "Stilpluralismus“, was die verfeindeten Positionen harmonisiert, oder man behandelt den einen oder den anderen - ohne die Methode der dialektischen Zusammenschau bzw. der Sicht auf kontrastierende Phänomene, die eine Zeit kulturell kristallisiert. Aber erst die Zusammensicht der Kontraste erhellt die Dimensionen einer Zeit. - Darin zeigt sich, wie wichtig (im Sinne der "Hermeneutik") für das Verstehen der heutigen zerrissenen Kunstlage schon allein die Vorauswahl des zu behandelnden Gegenstandes ist! Bereits 1923 hatte Carl Einstein, der es wissen mußte, geschrieben: „Die Pole heutiger Kunst liegen zum Reissen gespannt [...]“. (O. Dix, Das Kunstblatt [1923], 97 f.) 
schlechts-Feindlichkeit, und er setzt dem das Gegenteil entgegen: mit Stendhal pocht Nietzsche auf eine andere Wirkung des Schönen, nämlich als „une promesse de bonheur". Das Desinteresse Kants wird verworfen zugunsten des wirklichen "Artisten“ Stendhal (De l'amour, 1822). Wie Nietzsche das Ziel der Willensphilosophie perspektivisch von der Verneinung zur Bejahung des Lebens umkehrte, so kehrt er hier komplementär in der Perspektive seines Systems die Richtung um für das Problem des Ursprungs von Kunst in „Art und Grad von Geschlechtlichkeit“ des Individuums, d. h. im Charakter des Künstlers und in der Bedeutung der Kunst als „Stimulans des Lebens“, ja als „eigentliche metaphysische Tätigkeit dieses Lebens" (1872, Vorwort an R. Wagner). ${ }^{35}$

Daß der Mensch Kunst macht, daß er also des Kunstmachens bedürftig ist, kann nicht „ohne Interesse“ erfolgen und bleiben. Statt der willen-kalmierenden Wirkung des KunstSchönen (Schopenhauer) setzt Nietzsche die willen-erregende Wirkung von Schönheit und Künsten, das Interesse, die Erregung (Stendhal), das Ziel mithin der Lebenssteigerung. Nur so scheint ihm Kunstschaffen zutiefst evident zum Leben. Signifikanterweise erwähnte Nietzsche in dieser Passage der Genealogie en passant als Exemple die mythische Gestalt Pygmalions. ${ }^{36}$

Es wird somit im Ganzen m. E. deutlich, daß es bei diesem fundamentalen, vitalistischen Kunstbegriff letztlich kein "L'art pour l'art" geben kann, wenn die Kunst existentiell fundiert ist - vielmehr nur ein „L'art pour la vie“. Nietzsche kannte freilich Schlagwort und Ziele von jenem, setzte sich in der Fröhlichen Wissenschaft und in Götzendämmerung (Streifzüge 24) damit auseinander, aber verhöhnte es schließlich zutiefst: „L'art pour 1'art vielleicht: das virtuose Gequak kaltgestellter Frösche, die in ihrem Sumpfe desperieren ... Den ganzen Rest schuf die Liebe ..."37

Damit meinte Nietzsche, daß alle Kunst „lobt“, „verherrlicht“, , auswählt“, ja Wertschätzungen „stärkt oder schwächt“, d. h. letztlich: geht der Instinkt des echten Künstlers (nicht des Machers von Salonkunst oder des Scharlatans) auf die Kunst? „oder nicht vielmehr auf den Sinn der Kunst, das Leben? auf eine Wünschbarkeit von Leben? - Die Kunst ist das große Stimulans zum Leben. “ (GD, Streifzüge 24) ${ }^{38}$

35 GM, 3. Abh., No. 6, KGW VI/2, 364-367. Diese wichtige Passage für Nietzsches Kritik an Schopenhauer hat nicht genutzt W. Kaufmann, Nietzsche (dt. Ausg.), 154 f., der sogar fälschlich von einer „Schwachstelle“ in der Theorie der ästhetischen Werte bei Nietzsche sprach (155). Zwar erkannte Kaufmann das „vitale Interesse“, schöpfte aber Nietzsches Rekurs auf Stendhals Liebes-Begriff und dessen Formel "promesse de bonheur" nicht aus bzw. vermittelte sie nicht. Vgl. dagegen C. Türcke, Der tolle Mensch, 90-98.

36 Letztlich hängt das menschliche Kunstschaffen mit dem menschlichen „Trieb zur Metaphernbildung“ zusammen, die dem Menschen - bei seinem Todesbewußtsein - im Grunde erst ermöglicht zu leben, das Leben ermöglicht, es sinnstiftend macht: aus dem Willen zur Selbsterhaltung entspringen die Metaphern, der Mythos, die Kunst als quasi höhere Lebensformen (Über Wahrheit und Lüge im außermoralischen Sinne, in: Werke, Bd. III, hg. v. K. Schlechta, Darmstadt 1973, 319). Darauf hat jüngst C. Türcke überzeugend hingewiesen (Der tolle Mensch 53, 98), der dabei Nietzsches Wissen um seine Arten der Perspektiven-Umstellung (Ecce homo) betonte. Für den Kontext ist Pygmalion tatsächlich nicht nur eine Bildhauerfabel, sondern mehr, das Paradigma der bildenden Künste (vgl. dazu auch J. G. Herder, Plastik, Riga 1778; D. Schubert, Die Kunst Lehmbrucks, 85 f.).

37 NF von ca. April 1888, KGW VIII/3, 91 (14/120).

38 Vgl. dazu besonders die Beiträge von V. Gerhardt (zit. in Anm. 15) und W. Schulz, „Funktion und Ort der Kunst in Nietzsches Philosophie“, in: Nietzsche-Studien 12 (1983), 1-31. 
Diese umfassende Begründung seines Kunstverständnisses brachte Nietzsche 1886 in seinem Kommentar („Versuch einer Selbstkritik“) zur Geburt der Tragödie auf die Formel "Artisten-Metaphysik“ und im Jahr 1888 auf die Formel vom "Artisten-Evangelium“ (im Grunde das in Anm. 12 zitierte Programm der Aufgaben der Kunst für den Schaffenden $)^{39}$ - Kunstschaffen als die höchste Aufgabe und die eigentlich metaphysische Tätigkeit dieses Lebens.

39 Sein Artistenevangelium, sein Glaubensbekenntnis nennt Nietzsche diesen Brennpunkt seiner Philosophie (KGW VIII/3, 319 f.), vor welchem Hintergrund „der Wille zum Werden und Wechsel“ höher angesetzt wird als der zur "Wahrheit“; vgl. dazu W. Gebhard, Nietzsches Totalismus, $70 \mathrm{ff}$. 